\title{
Long-term mesh complications and reoperation after laparoscopic mesh sacrohysteropexy: a cross-sectional study
}

\author{
Matthew L. Izett-Kay ${ }^{1,2}$ (D) Dana Aldabeeb ${ }^{2} \cdot$ Anthony S. Kupelian $^{1} \cdot$ Rufus Cartwright $^{3} \cdot$ Alfred S. Cutner $^{1}$. \\ Simon Jackson ${ }^{3} \cdot$ Natalia Price $^{3} \cdot$ Arvind Vashisht $^{1,2}$
}

Received: 13 February 2020 / Accepted: 12 June 2020 / Published online: 3 July 2020

(C) The Author(s) 2020

\begin{abstract}
Introduction and hypothesis The paucity of long-term safety and efficacy data to support laparoscopic mesh sacrohysteropexy is noteworthy given concerns about the use of polypropylene mesh in pelvic floor surgery. This study is aimed at determining the incidence of mesh-associated complications and reoperation following this procedure.

Methods This was a cross-sectional postal questionnaire study of women who underwent laparoscopic mesh sacrohysteropexy between 2010 and 2018. Potential participants were identified from surgical databases of five surgeons at two tertiary urogynaecology centres in the UK. The primary outcome was patient-reported mesh complication requiring removal of hysteropexy mesh. Secondary outcomes included other mesh-associated complications, reoperation rates and Patient Global Impression of Improvement (PGI-I) in prolapse symptoms. Descriptive statistics and Kaplan-Meier survival analyses were used. Results Of 1,766 eligible participants, 1,121 women responded (response proportion 63.5\%), at a median follow-up of 46 months. The incidence of mesh complications requiring removal of hysteropexy mesh was $0.4 \%$ ( 4 out of 1,121$)$. The rate of chronic pain service use was $1.8 \%$, and newly diagnosed systemic autoimmune disorders was $5.8 \%$. The rate of reoperation for apical prolapse was $3.7 \%$, and for any form of pelvic organ prolapse it was $13.6 \%$. For PGI-I, $81.4 \%$ of patients were "much better" or "very much better".

Conclusions Laparoscopic mesh sacrohysteropexy has a low incidence of reoperation for mesh complications and apical prolapse, and a high rate of patient-reported improvement in prolapse symptoms. With appropriate clinical governance measures, the procedure offers an alternative to vaginal hysterectomy with apical suspension. However, long-term comparative studies are still required.
\end{abstract}

Keywords Laparoscopy $\cdot$ Pelvic organ prolapse $\cdot$ Reoperation $\cdot$ Surgical mesh $\cdot$ Uterine prolapse

26th Annual Scientific Meeting, United Kingdom Continence Society, Manchester, UK, 24-26th April 2019; Annual Scientific Meeting, British Society of Gynaecological Endoscopy, Newport, UK, 21-23 May 2019; IUGA/AUGS ASM, Nashville, USA, 24th to 28 September 2019

Electronic supplementary material The online version of this article (https://doi.org/10.1007/s00192-020-04396-0) contains supplementary material, which is available to authorized users

Matthew L. Izett-Kay

m.izett@ucl.ac.uk

1 Urogynaecology and Pelvic Floor Unit, University College London Hospitals, Clinic 2, Lower Ground Floor, EGA Wing, 235 Euston Road London NW12BU UK

2 UCL EGA Institute for Women's Health, University College London, Medical School Building, 74 Huntley Street London WC1E 6AU UK

3 Department of Urogynaecology, John Radcliffe Hospital, Oxford University Hospitals, Headley Way Oxford Headington OX3 9DU UK

\section{Introduction}

A recent systematic review has highlighted a lack of highquality data supporting mesh-augmented uterine-preserving surgical approaches for the treatment of uterine prolapse [1]. In the UK, the preferred procedure for the treatment of this highly prevalent condition is a vaginal hysterectomy $(\mathrm{VH})$ and apical suspension procedure, with or without concomitant colporrhaphy [2, 3]. However, this approach is limited by a high risk of recurrent vault prolapse, rates of reoperation for prolapse are between $4.6 \%$ and $18 \%[4,5]$. More importantly, a significant proportion of women would prefer uterine preservation if given the option [6]. These two factors may explain the growing use of uterine-sparing techniques [7].

Laparoscopic mesh sacrohysteropexy is one such uterinesparing procedure and involves re-suspending the uterus with polypropylene mesh anchored to the sacral promontory. 
Techniques are still evolving, but as undertaken in this study, the mesh was passed through two windows in the broad ligaments and anchored as a loop around the cervix. We have previously described this technique, as illustrated in Figure ESM 1 [8]. Reported advantages of laparoscopic sacrohysteropexy include lower blood loss and postoperative pain, longer vaginal length, and higher apical suspension compared with hysterectomy [1]. Our previously reported shortterm data showed high rates of symptom relief and low complication rates at 3-month follow-up in over 500 women following this procedure [9]. Additionally, we reported longer term outcomes of 110 patients at an average of 2.6 years postoperatively, corroborating the findings of high satisfaction and low rates of reoperation for pelvic organ prolapse (POP) [10].

The paucity of quality evidence for such procedures is significant given the controversies surrounding implantation of pelvic mesh. Transvaginal mesh for prolapse has been recognised as having high rates of complications and there remain concerns amongst patients around transvaginal mesh use for stress urinary incontinence (SUI) [11, 12]. Uterine preservation may confer the benefit of a lower risk of mesh erosion than that seen in other abdominal approaches to prolapse. Following subtotal hysterectomy and cervicopexy, the reported mesh exposure rate is between $4.3 \%$ and $10.5 \%$ [13]. There have been multiple national and international reviews of mesh and in some countries pelvic mesh remains partially or completely restricted from use [14-18]. Although mesh complications may be asymptomatic, they are often associated with chronic pain, leading to devastating impacts on quality of life [19]. Despite quality evidence refuting an association between systemic autoimmune disorders and mesh, some patients continue to worry about a possible link [20]. Regulators and reports into mesh have repeatedly identified a need for high-quality long-term cohort studies of pelvic mesh surgery $[21,22]$. On the basis of our clinical experience over the last decade we hypothesised the rate of these mesh-associated complications to be very low.

The aim of this study was to determine the rates of reoperation and mesh-associated complications in women who had undergone laparoscopic mesh sacrohysteropexy.

\section{Materials and methods}

We conducted a multicentre cross-sectional questionnaire study of women who underwent laparoscopic mesh sacrohysteropexy between February 2007 and September 2018, a timeframe from when the procedure was routinely offered until the start of this study. Potential participants were identified from the operating databases of five consultant surgeons based at two tertiary urogynaecology centres in the UK (University College London Hospitals, London and Oxford University Hospitals, Oxford), using operating procedure codes (OPCS) Y75.2 (laparoscopic approach to the abdominal cavity) or T43.9 (unspecified diagnostic endoscopic examination of the peritoneum) in combination with Q54.1 (suspension of the uterus NEC), Q54.4 (suspension of the uterus using mesh) or Q54.5 (sacrohysteropexy).

We included all English-speaking women over the age of 18 who underwent laparoscopic mesh sacrohysteropexy at one of our participating centres utilising the technique previously described, with steps illustrated in Figure ESM 1 [8]. This involves the use of a bifurcated polypropylene mesh wrapped around the cervix through broad ligament windows and secured anteriorly with non-absorbable sutures (Ethibond Excel $^{\mathrm{TM}}$; Ethicon) that is then secured to the sacral promontory with a helical fastener (Protack ${ }^{\mathrm{TM}}$; United States Surgical, Tyco Healthcare, Norwalk, CT, USA). According to local procurement policies the mesh used was either PRO-Lite ${ }^{\mathrm{TM}}$ (Atrium Medical Corporation, Hudson, NH, USA) or Prolene $^{\mathrm{TM}}$ mesh (Ethicon, Somerville, NJ, USA). The mesh used for individual participants was not available.

We excluded any patient who underwent a previous or subsequent trans-vaginal mesh-augmented prolapse operation, or concurrent mesh rectopexy and did not contact or include patients who were identified as deceased on our hospital databases.

The questionnaire used was designed to appropriately capture the outcome measures defined below and is contained in Appendix 1. Owing to the rare nature of many of the study's main outcomes, it was not possible to formally validate or test the reliability of the questionnaire prior to commencing the study. The questionnaire items were developed by the senior authors and then piloted at one site. This involved completion by women fulfilling our study inclusion criteria, who provided written or verbal feedback with respect to question comprehensibility. Potential participants were then contacted by post and able to respond by post in a prepaid envelope, or request a telephone questionnaire. Alternatively, they could submit responses using the REDCap electronic data capture tools hosted University College London [23, 24]. Research Electronic Data Capture (REDCap) is a secure, web-based software platform designed to support data capture for research studies, providing:

1. An intuitive interface for validated data capture

2. Audit trails for tracking data manipulation and export procedures

3. Automated export procedures for seamless data downloads to common statistical packages

4. Procedures for data integration and interoperability with external sources

Telephone interviews for the questionnaire were carried out according to a telephone script following verbal consent. Potential participants who had not responded to the 
first postal contact within 8 weeks were sent a second questionnaire.

Our study protocol was registered with the UK's Health Research Authority (HRA) and received a favourable research ethics committee (REC) opinion from the London City \& East REC on 11/05/2018 (reference 18/LO/0637), and favourable HRA approval. Participants gave consent to allow the study team to contact clinicians who managed any mesh complications, to obtain further details.

The primary outcome for our study was patient-reported mesh complications requiring the removal of the hysteropexy mesh. Patients were also asked the nature and timing of symptoms that led to the diagnosis of this mesh-associated complication. Secondary outcomes included the use or expectant use of chronic pain services, and the new diagnosis of a systemic autoimmune disorder (see Appendix 1). Further secondary outcomes included subsequent reoperation for POP and type of procedure, reoperation for SUI, Patient Global Impression of Improvement in prolapse symptoms (PGI-I prolapse) and the "Friends and Family test", asking whether participants would recommend the surgery if undergoing treatment for the same condition. All reported results come from the patient-reported data contained within the questionnaire responses, with the exception of the case details of those patients who reported reoperation for a mesh complication. In these nine women, case notes were obtained where available and with consent, for clarification of the nature of their reported mesh-associated complication.

Available data were analysed using descriptive statistics, with frequencies expressed as percentages. Survival analyses for mesh excision and reoperation for POP as the failure variables were undertaken using the Kaplan-Meier method. These analyses were performed using Stata/SE $15{ }^{\circledR}$ (StataCorp, College Station, TX, USA).

\section{Results}

We identified 1,766 potential participants, and following two rounds of postal contact, 1,121 women responded (response proportion 63.5\%), as shown in Fig. 1. The median length of follow-up from index hysteropexy was 46 months (range 2141 months), shown in Fig. 2. The average age of participants at the time of surgery was 58 years (range 24-86 years), other patient demographic details were unavailable owing to the nature of the surgical databases.

\section{Primary outcome}

The incidence of patient-reported mesh complications requiring removal of hysteropexy mesh, confirmed by case note review, was $0.4 \%$ ( 4 out of 1,121 ). This equated to 0.86 mesh removal operations per 1,000-person years of follow-up.
Figure 3 illustrates the Kaplan-Meier survival analysis, with patient-reported mesh complication requiring removal of the hysteropexy mesh as the failure variable. All reoperations for patient-reported mesh complications were undertaken within 4 years of the sacrohysteropexy. Details of the four cases in which participants reported mesh excision surgery are shown in Table 1.

Five patients reporting sacrohysteropexy mesh removal surgery were not included in the reporting of our primary outcome. One participant had undergone a concurrent synthetic mid-urethral sling for urinary incontinence at the time of sacrohysteropexy. She presented 18 months postoperatively with vaginal pain and dyspareunia and was found to have a small exposure of sub-urethral mesh. Partial excision of the sub-urethral portion of the tape from a vaginal approach was undertaken and the hysteropexy mesh was left in situ. She was followed up for 3 months and subsequently discharged. A second participant complained of abdominal pain within 4 weeks of surgery and was found to have haematometra. She underwent laparoscopic subtotal hysterectomy; the mesh was left in situ and used to undertake a stump cervicopexy. She was subsequently discharged from follow-up. The final 3 patients reporting reoperation for a mesh-associated complication had undergone reoperation for recurrent prolapse, 2 requiring mesh plication and the third opting for a vaginal hysterectomy.

\section{Secondary outcomes}

With respect to symptoms leading to removal of mesh, 2 participants reported pain and 2 reported bladder symptoms, as detailed in Table 2. Three women reported noticing the symptoms associated with the mesh complication as having developed within 12 months of their hysteropexy; details of these patient-reported data are shown in Table 2.

For the other patient-reported mesh-associated complications included within this study, $1.8 \%$ of the study participants (20 out of 1,121) reported that they had previously been or were awaiting referral to chronic pain services for pain specifically attributed to the mesh. With respect to systemic autoimmune diseases, 5.8\% (65 out of 1,121) of participants reported a new diagnosis of such a condition subsequent to their laparoscopic mesh sacrohysteropexy.

The risk of subsequent reoperation for POP was $13.6 \%$ (152 out of 1,121 ), and for SUI it was $2.3 \%$ (26 out of 1121), with details shown in Table 3. With respect to PGI-I prolapse, $81.4 \%$ of participants (912 out of 1,121) reported their symptoms to be "very much better" or "much better", and $82.2 \%$ (921 out of 1,121) would recommend the procedure to a friend or family member with the same condition. 
Fig. 1 Flow chart of participant recruitment

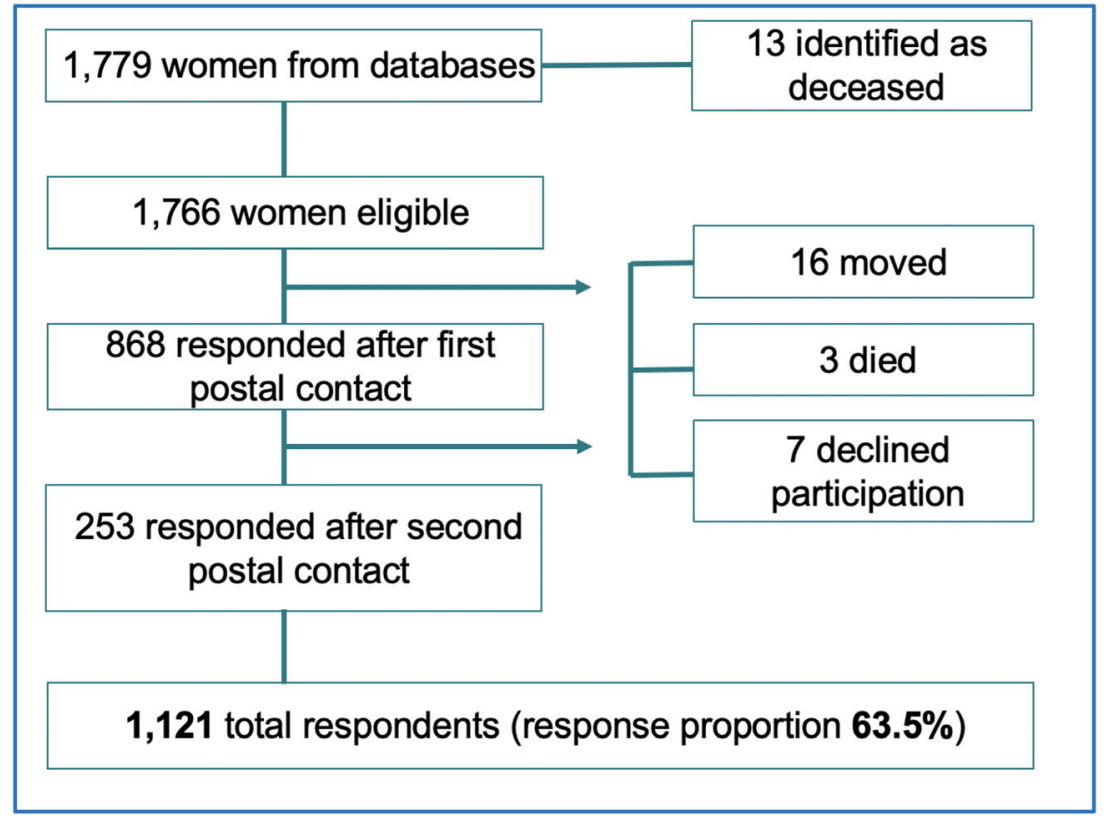

\section{Discussion}

\section{Main findings}

We report a low incidence of patient-reported mesh complications requiring mesh removal surgery confirmed by case note review, of $0.4 \%$ at a median follow-up of nearly 4 years, from a cohort of 1,121 women who underwent laparoscopic mesh sacrohysteropexy. Notably, there were no reported cases of vaginal mesh erosion.

This is the largest reported study of women who have undergone mesh-augmented uterine-preserving prolapse surgery, and the incidence of reoperation for a mesh-associated complication compares favourably with other gynaecological uses of mesh. For comparison, the risk of reoperation for a mesh complication following two of the most common such procedures, sacrocolpopexy and the synthetic mid-urethral sling, are $5 \%$ and $2.4 \%$ respectively $[25,26]$. According to the Cochrane review by Maher et al. [27] the risk of reoperation for mesh exposure following placement of transvaginal mesh for prolapse is $8 \%$, and in the PROSPECT trial, the largest randomised trial of transvaginal mesh, $4 \%$ of women required reoperation for a mesh complication $[12,27]$.

Comparison of our findings with the literature for sacrohysteropexy is difficult owing to the heterogeneity of reporting mesh complications. Long-term cohort studies have reported mesh erosion rates of $4 \%$ following robotics-assisted sacrohysteropexy, and 5\% following open sacrohysteropexy, with no details regarding reoperation [28, 29]. A variety of surgical techniques exist for sacrohysteropexy, with no clear
Fig. 2 Length of patient follow-

up

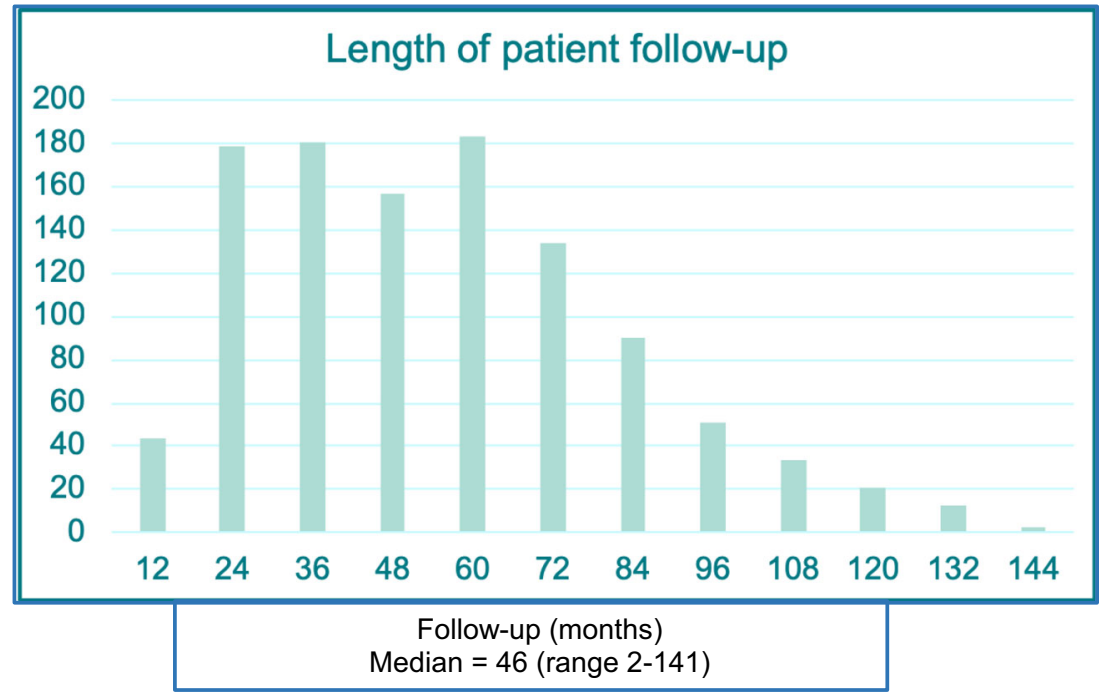


Fig. 3 Kaplan-Meier survival analysis with mesh removal surgery as the failure variable

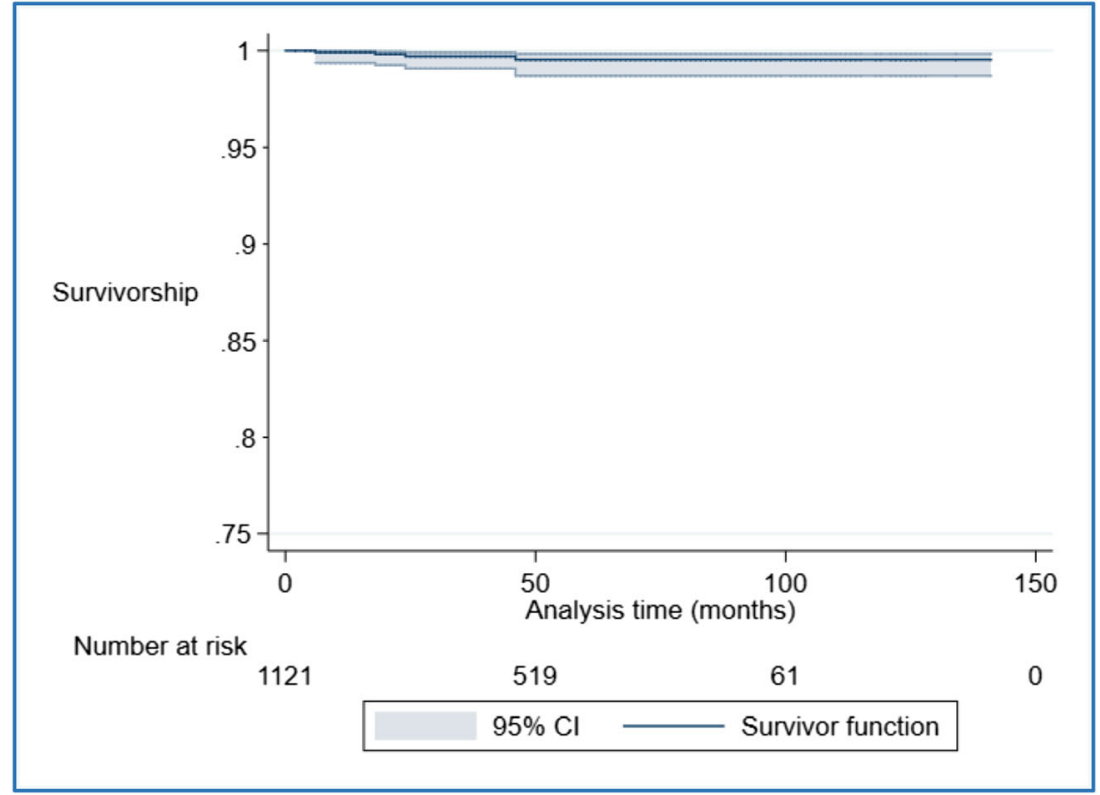

evidence to support one particular approach over another. Our technique differs from others reported in the literature in that there is limited dissection on the vagina, and mesh arms are sutured to the anterior cervix. It may be that the avoidance of mesh placement on the vagina explains the absence of vaginal mesh erosions at an average follow-up of nearly 4 years in contrast to the complications seen in these other series. Comparing mesh sacrohysteropexy and vaginal hysterectomy in a systematic review, Meriwether et al. did not report any cases of mesh erosion or reoperation following abdominal mesh sacrohysteropexy [1]. Most of the studies included in this part of the review either failed to report these outcomes, or they were only seen in the comparator groups undergoing hysterectomy and concurrent sacrocolpopexy, where the risk of reoperation for a mesh complication was between 2 and $3 \%$.

With respect to the other mesh-associated complications, the risk of utilising chronic pain services in our study appears relatively low; following vaginal hysterectomy, the risk of chronic pain may be as high as $25 \%$ [30]. Our finding of a $5.8 \%$ risk of subsequent diagnosis of a systemic autoimmune disorder is higher than the $2.8 \%$ risk reported by the largest and most methodologically robust available study looking at the association between gynaecological mesh and autoimmune disease [20]. However, in that matched cohort study, over $40 \%$ of women undergoing mesh-augmented POP surgery had pre-existing diagnoses of autoimmune conditions, and were not included in the analysis. We were unable to account for pre-existing autoimmune disease in our participants to allow for exclusion from our analysis, and therefore this finding should be interpreted with caution.

It is noteworthy that in our study only $3.7 \%$ of our patients underwent a subsequent apical prolapse procedure, compared with the $6-8 \%$ risk reported following vaginal hysterectomy
[31]. It is uncertain if this finding supports the role of mesh augmentation for repair of apical prolapse; the issue remains controversial. Reoperation rates for prolapse of any compartment in our study are comparable with the overall reoperation rate for POP following VH, which was estimated to be $11 \%$ at 5 years in a recent large Danish registry study [32]. It is significantly lower than reoperation rates following suture hysteropexy, which was reported to be $30 \%$ in the same study. The $9 \%$ risk of subsequent colporrhaphy in our study may reflect surgical practice within participating centres. Concurrent anterior and posterior compartment repair for prolapse above the hymenal ring is generally avoided, as evidence suggests that such prolapse might be less likely to be symptomatic, and could be considered normal [33]. The authors acknowledge that this may lead to higher rates of colporrhaphy at a later date; indeed, our own reoperation rates in this study are higher than those reported in our previous cohorts, likely a feature of the longer follow-up [9, 10]. Reoperation for SUI is a recognised risk of POP surgery; the incidence of this in our study is comparable to the $2 \%$ risk quoted in large national studies [34]. The PGI-I prolapse has been validated as a measure for POP surgery, and we report high rates of improvement in prolapse symptoms [35].

\section{Significance and implications}

The findings from our study are important given the large cohort, long-term follow-up, and importantly, the patientreported nature of our outcomes. Despite well-publicised controversies surrounding the use of mesh in pelvic floor surgery, the majority of patients would recommend laparoscopic mesh sacrohysteropexy to friends or family. This, in combination with a low risk of reoperation for mesh complications and low 
Table 1 Case details of patient-reported mesh complications

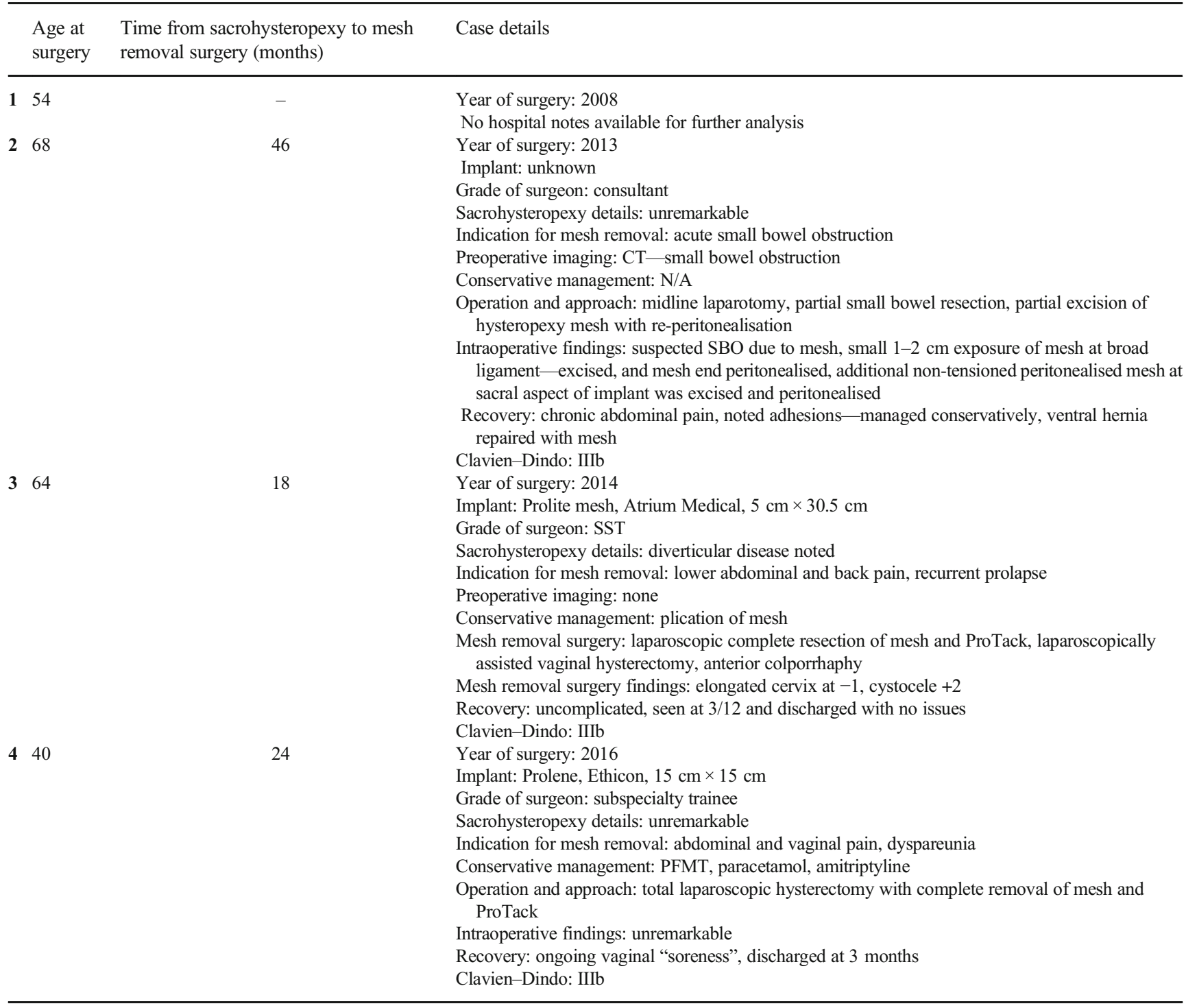

rates of chronic pain service use, should provide reassurance to both clinicians and women considering the procedure, as well as regulatory bodies.

The role of mesh-augmented prolapse surgery, specifically sacrohysteropexy, deserves ongoing scrutiny. Although the hierarchy of evidence-based medicine would favour the need for large, multicentre prospective studies, these are notoriously difficult for surgical interventions. Additionally, the controversies surrounding mesh in pelvic floor surgery are likely to make such studies difficult to undertake. Although large database studies would be helpful, researchers often depend on data from clinicians or hospital coders, who have their own issues with respect to accuracy. Therefore, such studies are valuable in providing a pragmatic evidence base for women considering surgery for POP, as well as clinicians and regulators.

\section{Strengths and limitations}

The principle strengths of our study include the long-term follow-up of a large number of participants, operated on by several surgeons at different institutions using a standardised follow-up in the form of a questionnaire. The value of patient-reported data cannot be understated given the current climate surrounding mesh use, with anecdotal concerns about a potential breakdown in trust between patients and their clinicians [36].

There are methodological limitations inherent within our study design, such as a lack of a comparison group, the use of patient-reported data without routine clinical or case note review to confirm the reported outcomes, and a paucity of demographic data to allow for regression analysis. Owing to the rare nature of our primary outcome, the use of a validated patient-reported outcome measure questionnaire was not 
Table 2 Patient- reported events leading to mesh complication

\begin{tabular}{ll}
\hline Parameter & Data, $n(\%)$ \\
\hline Reason for mesh removal & - \\
Asymptomatic & - \\
Pain on examination & - \\
Pain during sex & $1(25 \%)$ \\
Pain during physical/daily activities & $1(25 \%)$ \\
Pain unrelated to above & - \\
Vaginal discharge & $2(50 \%)$ \\
Bladder symptoms & - \\
Bowel symptoms & - \\
Timeframe from operation to symptoms of mesh complication \\
$<48$ h & $1(25 \%)$ \\
49 h to 2 months & $2(50 \%)$ \\
3 months to 12 months & $1(25 \%)$ \\
$>12$ months &
\end{tabular}

possible. Study participants underwent their procedures in centres with specific expertise in laparoscopic pelvic floor surgery, and therefore the research setting and findings may not be applicable to other centres. As with all questionnaire studies, results are affected by recall and response bias of participants. Participants may well not recall undergoing a subsequent surgical procedure or the nature of such surgery. Consideration of these biases also applies to the interpreting the use of chronic pain services, and subsequent diagnosis of a systemic autoimmune disorders. Finally, owing to the lack of demographic data, we cannot comment on how representative our participants are of the whole cohort of potential participants. Those greatly troubled by mesh complications may have opted not to participate or, conversely, were keenest to respond. Similarly, our subjective outcomes of PGI-I prolapse and the "friends and family" test may be adversely influenced by mesh concerns and media coverage.

Table 3 Subsequent procedures for pelvic organ prolapse $(P O P)$ and stress urinary incontinence (SUI)

\begin{tabular}{lc}
\hline Parameter & Data, $n(\%)$ \\
\hline Subsequent POP procedure $(N=152)$ & \\
Apical procedure & $41(3.7 \%)$ \\
Hysterectomy & $9(0.8 \%)$ \\
Colporrhaphy & $102(9.1 \%)$ \\
Subsequent SUI procedure $(N=26)$ & \\
Synthetic mid urethral sling & $13(1.2 \%)$ \\
Mid urethral fascial sling & $1(0.1 \%)$ \\
Periurethral bulking & $3(0.4 \%)$ \\
Colposuspension & $5(0.5 \%)$ \\
Unspecified & $4(0.4 \%)$ \\
\hline
\end{tabular}

\section{Conclusion}

This study provides a large, patient-reported dataset offering a pragmatic insight with respect to the wider context of other forms of available evidence on the safety and efficacy of mesh-augmented sacrohysteropexy. In our opinion, the low risk of reoperation for a mesh-associated complication provides reassurance that laparoscopic mesh sacrohysteropexy can continue to be offered with appropriate decision-making processes, consent, clinician training and audit.

Contributions Matthew Izett-Kay: project development, data collection, manuscript writing; Dana Aldabeeb: data collection, manuscript writing; Anthony Kupelian: project development, manuscript writing; Rufus Cartwright: project development, manuscript writing; Alfred Cutner: project development, manuscript writing; Simon Jackson: project development, manuscript writing; Natalia Price: project development, manuscript writing; Arvind Vashisht: project development, manuscript writing.

Funding Consumables and administrative costs: Elizabeth Garrett Anderson Charity, UK, and the British Society for Gynaecological Endoscopy (BSGE), Travelling Fellowship.

\section{Compliance with ethical standards}

Conflicts of interest Matthew Izett-Kay: none; Dana Aldabeeb: none; Anthony Kupelian: honorarium from Olympus; Rufus Cartwright: none; Alfred Cutner: honoraria from Olympus, Stryker and consultancy to Fannin and Coloplast; Simon Jackson: none; Natalia Price: none; Arvind Vashisht: honoraria from Olympus and BARD Medical.

Open Access This article is licensed under a Creative Commons Attribution 4.0 International License, which permits use, sharing, adaptation, distribution and reproduction in any medium or format, as long as you give appropriate credit to the original author(s) and the source, provide a link to the Creative Commons licence, and indicate if changes were made. The images or other third party material in this article are included in the article's Creative Commons licence, unless indicated otherwise in a credit line to the material. If material is not included in the article's Creative Commons licence and your intended use is not permitted by statutory regulation or exceeds the permitted use, you will need to obtain permission directly from the copyright holder. To view a copy of this licence, visit http://creativecommons.org/licenses/by/4.0/.

\section{References}

1. Meriwether KV, Antosh DD, Olivera CK, et al. Uterine preservation vs hysterectomy in pelvic organ prolapse surgery: a systematic review with meta-analysis and clinical practice guidelines. Am J Obstet Gynecol. 2018;219(2):129-146.e2.

2. Jha S, Cutner A, Moran P. The UK national prolapse survey: 10 years on. Int Urogynecol J. 2018;29(6):795-801.

3. Tegerstedt G, Maehle-Schmidt M, Nyrén O, Hammarström M. Prevalence of symptomatic pelvic organ prolapse in a Swedish population. Int Urogynecol J. 2005;16(6):497-503.

4. Jelovsek JE, Barber MD, Brubaker L, et al. Effect of uterosacral ligament suspension vs sacrospinous ligament fixation with or without perioperative behavioral therapy for pelvic organ vaginal prolapse on surgical outcomes and prolapse symptoms at 5 years in 
the OPTIMAL randomized clinical trial. JAMA. 2018;319(15): 1554-65.

5. Edenfield AL, Amundsen CL, Weidner AC, Wu JM, George A, Siddiqui NY. Vaginal prolapse recurrence after uterosacral ligament suspension in normal-weight compared with overweight and obese women. Obstet Gynecol. 2013;121(3):554-9.

6. Frick AC, Barber MD, Paraiso MFR, Ridgeway B, Jelovsek JE, Walters MD. Attitudes toward hysterectomy in women undergoing evaluation for uterovaginal prolapse. Female Pelvic Med Reconstr Surg. 2013;19(2):103-9.

7. Zacche MM, Mukhopadhyay S, Giarenis I. Trends in prolapse surgery in England. Int Urogynecol J. 2018;29(11):1689-95.

8. Rahmanou P, Price N, Jackson S. Laparoscopic hysteropexy: a novel technique for uterine preservation surgery. Int Urogynecol J. 2014;25(1):139-40.

9. Jefferis H, Price N, Jackson S. Laparoscopic hysteropexy: 10 years' experience. Int Urogynecol J. 2017;28(8):1241-8.

10. Kupelian AS, Vashisht A, Sambandan N, Cutner A. Laparoscopic wrap round mesh sacrohysteropexy for the management of apical prolapse. Int Urogynecol J. 2016;27(12):1889-97.

11. Gornall J. How mesh became a four letter word. BMJ. 2018;363: $\mathrm{k} 4137$.

12. Glazener CM, Breeman S, Elders A, et al. Mesh, graft, or standard repair for women having primary transvaginal anterior or posterior compartment prolapse surgery: two parallel-group, multicentre, randomised, controlled trials (PROSPECT). Lancet. 2017;389(10067):381-92.

13. Jia X, Glazener C, Mowatt G, et al. Systematic review of the efficacy and safety of using mesh in surgery for uterine or vaginal vault prolapse. Int Urogynecol J. 2010;21(11):1413-31.

14. The safety of surgical meshes used in urogynaecological surgery. In. https://ec.europa.eu/health/scientific_committees/consultations/ public_consultations/scenihr_consultation_27_en: SCENIHR, European Commission; 2015.

15. Mesh Oversight Group Report. In. https://www.england.nhs.uk/ publication/mesh-oversight-group-report/: NHS England; 2017.

16. TGA undertakes regulatory actions after review into urogynaecological surgical mesh implants. https://www.tga.gov. au/alert/tga-actions-after-review-urogynaecological-surgicalmesh-implants: Therapeutic Goods Administration, Australian Department of Health; 2018.

17. Wise J. Surgical mesh for stress urinary incontinence to be halted immediately in England. BMJ. 2018;362:k3035.

18. The Scottish independent review of the use, safety and efficacy of transvaginal mesh implants in the treatment of stress urinary incontinence and pelvic organ prolapse in women: final report. In. www.gov. scot/Publications/2017/03/3336/3: Scottish Government; 2017.

19. Geller EJ, Babb E, Nackley AG, Zolnoun D. Incidence and risk factors for pelvic pain after mesh implant surgery for the treatment of pelvic floor disorders. J Minim Invasive Gynecol. 2017;24(1): $67-73$.

20. Chughtai B, Sedrakyan A, Mao J, Eilber KS, Anger JT, Clemens JQ. Is vaginal mesh a stimulus of autoimmune disease? Am J Obstet Gynecol. 2017;216(5):495.e1-7.
21. Chapple CR, Cruz F, Deffieux X, et al. Consensus statement of the European Urology Association and the European Urogynaecological Association on the use of implanted materials for treating pelvic organ prolapse and stress urinary incontinence. Eur Urol. 2017;72(3):424-31.

22. Urinary incontinence and pelvic organ prolapse in women: management [NG 123]. In: National Institute for Health and Care Excellence; 2019.https://www.nice.org.uk/guidance/ng123

23. Harris PA, Taylor R, Minor BL, et al. The REDCap consortium: building an international community of software platform partners. J Biomed Inform. 2019;95:103208.

24. Harris PA, Taylor R, Thielke R, Payne J, Gonzalez N, Conde JG. Research electronic data capture (REDCap) - a metadata-driven methodology and workflow process for providing translational research informatics support. J Biomed Inform. 2009;42(2):377-81.

25. Nygaard I, Brubaker L, Zyczynski HM, et al. Long-term outcomes following abdominal sacrocolpopexy for pelvic organ prolapse. JAMA. 2013;309(19):2016-24.

26. Keltie K, Elneil S, Monga A, et al. Complications following vaginal mesh procedures for stress urinary incontinence: an 8 year study of 92,246 women. Sci Rep. 2017;7(1):12015.

27. Maher C, Feiner B, Baessler K, Christmann-Schmid C, Haya N, Marjoribanks J. Transvaginal mesh or grafts compared with native tissue repair for vaginal prolapse. Cochrane Database Syst Rev. 2016;2(2):CD012079.

28. Grimminck K, Mourik S, Tjin-Asjoe F, Martens J, Aktas M. Longterm follow-up and quality of life after robot assisted sacrohysteropexy. E J Obstet Gynecol Reprod Biol. 2016;206:27-31.

29. Paek J, Lee M, Kim BW, Kwon Y. Robotic or laparoscopic sacrohysteropexy versus open sacrohysteropexy for uterus preservation in pelvic organ prolapse. Int Urogynecol J. 2016;27(4):593-9.

30. Brandsborg B, Nikolajsen L, Kehlet H, Jensen TS. Chronic pain after hysterectomy. Acta Anaesthesiol Scand. 2008;52(3):327-31.

31. Aigmueller T, Dungl A, Hinterholzer S, Geiss I, Riss P. An estimation of the frequency of surgery for posthysterectomy vault prolapse. Int Urogynecol J. 2010;21(3):299-302.

32. Husby KR, Larsen MD, Lose G, Klarskov N. Surgical treatment of primary uterine prolapse: a comparison of vaginal native tissue surgical techniques. Int Urogynecol J. 2019:;30(11):1887-93.

33. Dietz H, Mann K. What is clinically relevant prolapse? An attempt at defining cutoffs for the clinical assessment of pelvic organ descent. Int Urogynecol J. 2014;25(4):451-5.

34. Altman D, Granath F, Cnattingius S, Falconer C. Hysterectomy and risk of stress-urinary-incontinence surgery: nationwide cohort study. Lancet. 2007;370(9597):1494-9.

35. Srikrishna S, Robinson D, Cardozo L. Validation of the patient global impression of improvement (PGI-I) for urogenital prolapse. Int Urogynecol J. 2010;21(5):523-8.

36. Independent Medicines and Medical Devices Safety Review. http:// www.immdsreview.org.uk/, 2019.

Publisher's note Springer Nature remains neutral with regard to jurisdictional claims in published maps and institutional affiliations. 